\title{
Evaluadores que han participado en el presente número
}

\author{
Alberto Gómez Bautista (Centro de Lenguas e Culturas da Universidade de \\ Aveiro) \\ Antoni Ferrando (Universitat de València) \\ Carlos Caetano Vizcaíno (Universidade da Coruña) \\ Jaume Corbera (Universitat de les Illes Balears) \\ Jerardo Elortza (Mondragon Unibertsitatea) \\ Joan Mahiques (Universitat Jaume I) \\ Joaquim Martí (Universitat de València) \\ Lierni Munduate Oruesagasti (Universidad Complutense de Madrid) \\ Maria Josep Cuenca (Universitat de València) \\ Mikel Etxaburu (Mondragon Unibertsitatea) \\ Mikel Gartziarena (Euskal Herriko Unibertsitatea) \\ Patricia Arias Chachero (IES Fermín Bouza, Xunta de Galicia) \\ Simona Škrabec (Universitat Oberta de Catalunya) \\ Vicent Salvador Liern (Universitat Jaume I) \\ Vicente Huici Urmeneta (Deustuko Unibertsitatea) \\ Xavier Bonillo (Universitat Oberta de Catalunya)
}

